\title{
Construction of Circular Quadrature Amplitude Modulations (CQAM)
}

\author{
Johannes Van Wonterghem*, Joseph J. Boutros $\dagger$, and Marc Moeneclaey* \\ ${ }^{*}$ Dept. of Telecommunications and Information Processing, Ghent University, 9000 Ghent, Belgium, \\ \{johannes.vanwonterghem,marc.moeneclaey\}@ugent.be \\ ${ }^{\dagger}$ Dept. of Electrical and Computer Engineering, Texas A\&M University, 23874 Doha, Qatar, boutros@tamu.edu
}

\begin{abstract}
Circular quadrature amplitude modulations (CQAM) are introduced as an alternative to mono-dimensional ASK constellations (and their QAM Cartesian product) for probabilistic shaping with non-binary error-correcting codes. We propose an exact construction method via recursive equations for bidimensional CQAM constellations. We show that CQAMs are subsets of the hexagonal lattice $A 2$ for a particular alphabet size. Then we describe CQAM constructions in three dimensions from the D3 (fcc) lattice and from Fibonacci spirals.
\end{abstract}

\section{INTRODUCTION}

Quadrature Amplitude Modulations (QAM) are employed in almost all digital communication systems over the Internet, in cellular networks, in satellite links, in fiber optic links, and in wireless local area networks [11]. Most QAM constellations are carved from the bi-dimensional cubic lattice and have a square or a rectangular shape. The error rate performance of QAM constellations is dramatically improved with the use of powerful error-correcting codes. However, a high coding gain is not sufficient to achieve channel capacity. For additive white Gaussian noise channels, the input distribution should mimic a Gaussian distribution to approach capacity. Geometric shaping with equiprobable signaling is an excellent method to achieve capacity with real constellations [14] or complex constellations [9]. The Voronoi cell of a lattice is another means for shaping a constellation and achieving near-capacity performance [6]. Recent results on geometric shaping with a Gaussian-like codebook are very promising [3].

The Gaussian-like channel input can also be realized via a probabilistic shaping of the signal constellation [7] [1]. This paper deals with the construction of signal constellations for probabilistic shaping. Circular QAM were introduced in [2] to make probabilistic shaping feasible with non-binary codes without going back to the bit level. The next section gives a quick overview of probabilistic shaping and how CQAM fits non-binary coding. An exact construction method for bi-dimensional CQAM, called the triangular construction, is presented in Section III. Section III-B shows interesting connections between the hexagonal lattice and 2D CQAM. Similar connections are presented in Section IV between the fcc lattice and 3D CQAM. Section IV also shows how to build CQAM in three dimensions from Fibonacci spirals.

\section{PROBABILISTIC SHAPING FOR NON-BINARY CODES}

All types of digital transmission systems combining errorcorrecting codes and probabilistic shaping of the modulator constellation can be represented by the model depicted in Figure 1. It is assumed that the information source is uniform over a $q$-ary alphabet and the channel code is defined over a $p$-ary alphabet (a field or a ring), where $q \geq 2$ and $p=q^{m}$, $m \geq 1$. A distribution matcher is applied to a fraction or to all information symbols in order to generate new symbols with a Gaussian-like prior distribution. These non-uniform information symbols are shown in red in Figure 1 and their main role is to shape the amplitude of the signal constellation. Uniform symbols after encoding include parity symbols (the code redundancy) and potentially a fraction of those uniform information symbols from the source. Uniform symbols are not involved in probabilistic amplitude shaping and should be handled by the constellation mapping without perturbing the shaping scheme. A proof is given in Section II-B that justifies the assumption of uniform parity symbols after encoding over $G F\left(p^{m}\right)$ (prime $p$ ) or $\mathbb{Z} / p \mathbb{Z}$ (arbitrary $p$ ).

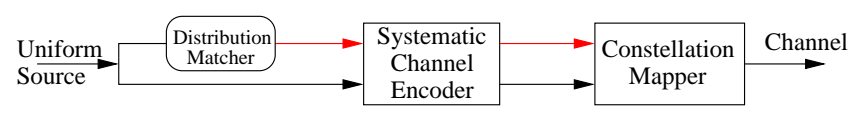

Figure 1. General system model for probabilistic amplitude shaping.

\section{A. From binary to non-binary coding}

For mono-dimensional constellations, uniform symbols are mapped to the sign of a constellation point. An illustration is given in Figure 2. This corresponds to $q=2$ and $p=2^{m}$, $m=1$ for binary codes [1] and $m>1$ for characteristic2-field non-binary codes [13]. Each 8-ASK point has a label of 3 bits. The two bits in red follow the prior distribution created by the distribution matcher (probability mass function represented by the red bars). On the other hand, parity bits are all assigned to the sign bit. Hence, for mono-dimensional constellations, the coding rate is taken to be larger than or equal to $\frac{\log _{2}(M)-1}{\log _{2}(M)}$, where $M$ is the constellation size.

Probabilistic amplitude shaping was generalized to complex constellations by introducing circular symmetry [2]. Similar to Hadamard transform with \pm generalized to Fourier transform with $e^{2 \pi \sqrt{-1} / p}$, the sign approach in mono-dimensional 


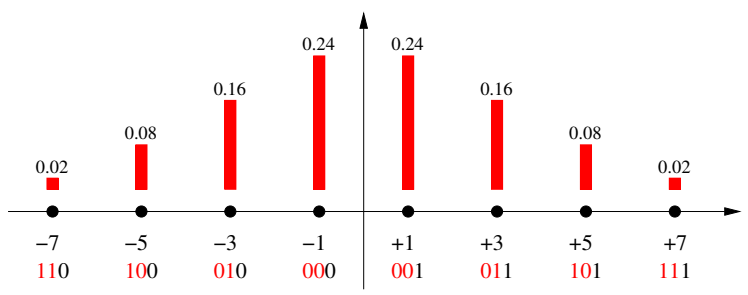

Figure 2. Probabilistic amplitude shaping of 8-ASK constellation.

constellations is replaced by a phase approach. A complex constellation suited to probabilistic amplitude shaping should have its points organized in circles around the origin: nonuniform symbols select a circle and uniform symbols select a point on the circle. The CQAM defined in [2] has $M=p^{2}$ points organized in $p$ circles (also called shells) and $p$ points per shell. Examples of CQAM are shown in Figures $3 \& 4$.

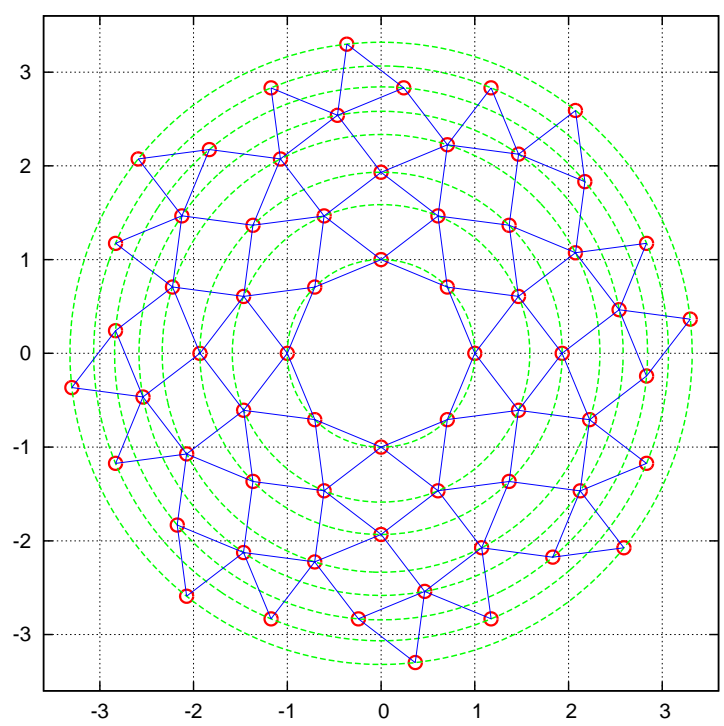

Figure 3. The 64 points of an $8^{2}$-CQAM constellation. Edges are connecting pairs of points located at minimum distance from each other.

For simplicity, it is assumed that the radius of the first shell, i.e. the inner radius of the CQAM, is $\rho_{0}=1$. The set of $p^{2}$ points of a CQAM shall be denoted by $\mathcal{A}=\left\{x_{k}\right\}_{k=0}^{p^{2}-1}$, where point $x_{i p+\ell}$ is the $\ell$ th point on shell $i, 0 \leq i, \ell \leq p-1$. The minimum Euclidean distance of CQAM is $d_{E \min }(\mathcal{A})=2 \sin \left(\frac{\pi}{p}\right)$. The average energy of CQAM is $E_{s}=\sum_{i=0}^{p-1} \pi_{i} \rho_{i}^{2}$, where $\pi_{i}$ is the a priori probability distribution of the amplitude as imposed by the distribution matcher. A CQAM point has a priori probability $\pi\left(x_{i p+\ell}\right)=\frac{\pi_{i}}{p}$. The average energy in the uniform case, when all points are equiprobable, becomes $E_{s}($ unif $)=\sum_{i=0}^{p-1} \rho_{i}^{2} / p$.

There exist infinitely many ways to place $p$ circles around the origin in the complex plane with $p$ points per circle. The original CQAM construction aimed at maximizing the figure of merit given by the ratio $\frac{d_{E \min }^{2}}{E_{s}(u n i f)}$. The construction algorithm from [2] can be summarized as follows:

- Original construction algorithm for 2D $p^{2}$-CQAM.

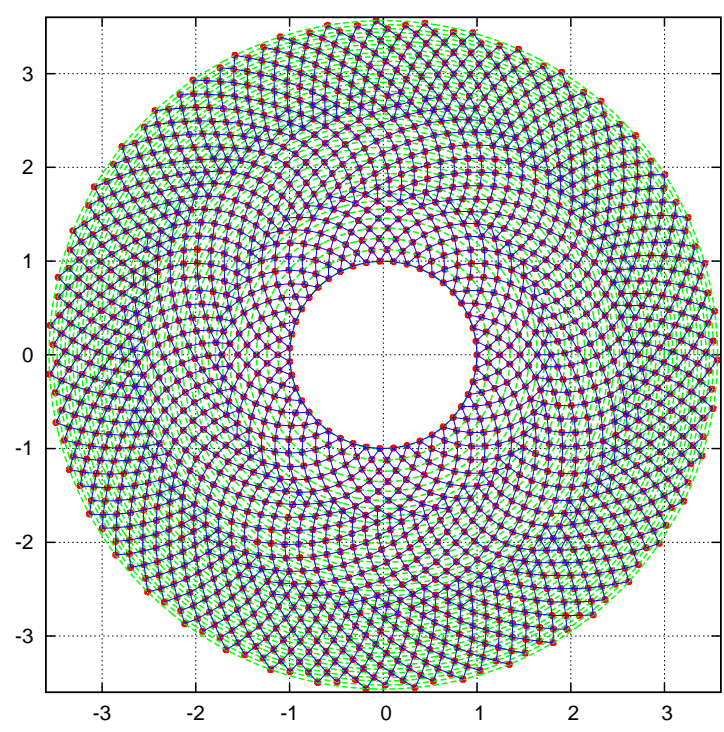

Figure 4. The 1849 points of $43^{2}$-CQAM constellation. Notice the presence of rings appearing at $\sqrt{3}$ and $\sqrt{7}$, while the outer radius approaches $\sqrt{13}$.

Given constellation points on shells 0 to $i-1$, put $p$ equidistant points on shell $i$ and numerically find the smallest radius $\rho_{i}$ for this shell, $\rho_{i} \geq \rho_{i-1}$, and the best phase shift $\phi_{i}$ such that $d_{\text {Emin }}$ is satisfied.

The drawbacks of the original CQAM construction algorithm are: 1- Slow construction for large $p$. 2- Propagation of numerical errors. 3- No explanation for the waves defining three ring zones and the outer radius limit. In Section III describing the exact triangular construction, we will prove that the three CQAM ring zones are separated by radii $\sqrt{3}$ and $\sqrt{7}$. The reader may check that the outer radius is bounded from above by $\sqrt{13}$ via the recursive equations of Theorem 5 .

\section{B. Uniformity of parity symbols in fields and rings}

Lemma 1 and Theorem 1 in [2] prove that parity symbols obtained from linear encoding over a prime field $\mathbb{F}_{p}$ with a dense generator matrix tend to have a uniform distribution. The next theorem generalizes the result to any field. The integers $q$ and $p$ below should not be confused with $q$ and $p$ used earlier in this section to define the source alphabet size and the code alphabet size.

Theorem 1: Let $\mathbb{F}_{q}$ be a finite field, where $q=p^{m}$ with $p$ prime and $m \geq 1$. Consider a sequence $\left\{s_{\ell}\right\}_{\ell \geq 1}$ of independent random symbols over $\mathbb{F}_{q}$. Suppose that the probability distributions of $\left\{s_{\ell}\right\}_{\ell \geq 1}$ satisfy

$\liminf \operatorname{in}_{\ell \rightarrow \infty}\left\{\min _{u \in \mathbb{F}_{q}} \operatorname{Pr}\left\{s_{\ell}=u\right\}\right\}>0$. Then

$$
\forall \gamma \in \mathbb{F}_{q}, \quad \lim _{k \rightarrow \infty} \operatorname{Pr}\left(\sum_{\ell=1}^{k} s_{\ell}=\gamma\right)=1 / q .
$$

Proof: Let $S=\sum_{\ell=1}^{k} s_{\ell}$ and $q_{\ell}(u)=\operatorname{Pr}\left\{s_{\ell}=u\right\}$. Using techniques similar to Theorem 1 of [2], firstly we prove the 
following

$$
\operatorname{Pr}(S=\gamma)=\frac{\left(1+\sum_{v} \prod_{\ell=1}^{k}\left(\sum_{u} q_{\ell}(u) \omega^{<u+\gamma, v>}\right)\right)}{q},
$$

where $u$ spans all elements of $\mathbb{F}_{q}, v$ spans all non-zero elements of $\mathbb{F}_{q}, \omega=\exp (2 \pi \sqrt{-1} / p)$ is a $p$-th root of unity, and $\langle u, v\rangle$ denotes the scalar product of two elements from $\mathbb{F}_{q}$ seen as two vectors $\left(\mathbb{F}_{q}\right.$ is a vector space of dimension $m$ over $\mathbb{F}_{p}$ ). The lim inf condition on the distribution $\operatorname{Pr}\left\{s_{\ell}=u\right\}$ guarantees that $\left|\sum_{u} q_{\ell}(u) \omega^{<u+\gamma, v>}\right|<1$ so the right term in the numerator of the expression of $\operatorname{Pr}(S=\gamma)$ vanishes.

The result of Theorem 1 can be easily extended to any ring using a similar proof. The liminf condition is mandatory to keep the weighted sum of powers of $\omega$ inside the unit circle, because divisors of zero in the ring are going to create many identical powers of $\omega$ within that sum.

Theorem 2: Let $p$ be an arbitrary positive integer, $p \geq 2$. Consider a sequence $\left\{s_{\ell}\right\}_{\ell \geq 1}$ of independent random symbols over the ring $\mathbb{Z}_{p}=\mathbb{Z} / p \mathbb{Z}$,

where $\liminf \operatorname{in}_{\ell \rightarrow \infty}\left\{\min _{u \in \mathbb{Z}_{p}} \operatorname{Pr}\left\{s_{\ell}=u\right\}\right\}>0$. Then

$$
\forall \gamma \in \mathbb{Z}_{p}, \quad \lim _{k \rightarrow \infty} \operatorname{Pr}\left(\sum_{\ell=1}^{k} s_{\ell}=\gamma\right)=1 / p .
$$

\section{TRiangular CONSTRUCTION OF 2D CQAM}

The exact construction of a CQAM constellation deals with tiling equilateral and isosceles triangles on top of each others starting from the unit circle. We shall call it the triangular construction. The reader can observe such tiling of triangles in Figure 3.

\section{A. Establishing three ring zones and the construction}

By convention we took $\rho_{0}=1$. On the first shell, also by convention, the first point has phase $\phi_{0}=0$. The remaining $p-1$ points on this shell are determined by successive rotations over an angle of $\frac{2 \pi}{p}$. Now, the construction of the second shell is straightforward: Just draw equilateral triangles with the bases defined by consecutive points on shell 0 . The outer corners of the equilateral triangles form the second shell $(i=1)$ with $\phi_{1}=\frac{\pi}{p}$.

On any upper shell $i$, after finding its radius $\rho_{i}$ and the phase $\phi_{i}$ of its first point, rotating by $\frac{2 \pi}{p}$ gives the remaining $p-1$ points. The main constraint while building shell $i$ is to minimize $\rho_{i}$ while maintaining the minimum Euclidean distance $d=d_{\operatorname{Emin}}(\mathcal{A})$ with lower shells. Let us consider three consecutive shells $i, i-1$, and $i-2$ as shown in Figure 5 .

Let $A$ and $B$ be the length of two line segments as drawn in the figure. We have $\frac{d}{2} \leq A=\sqrt{d^{2}-B^{2}}$ and $2 B \leq d \sqrt{3}$. But $B=\rho_{i-1} \sin \left(\frac{\pi}{p}\right)$ which yields $\rho_{i-1} \leq \sqrt{3}$. This defines a first ring zone for all CQAM shells with radii in the range $[1, \sqrt{3}]$.

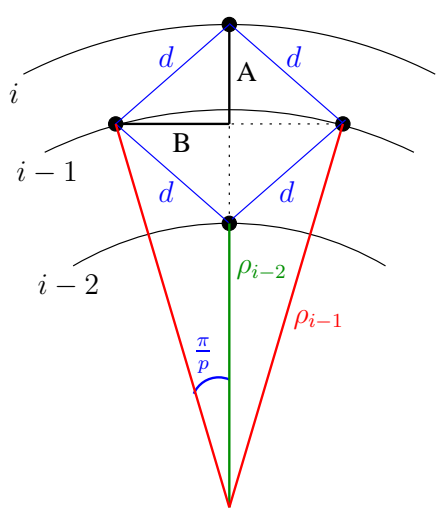

Figure 5. Representation of points in three shells in ring zone 1.

Also, from the representation of the three shells, it is easy to prove that $\rho_{i}=\rho_{i-2}+d \cdot \sqrt{4-\rho_{i-1}^{2}}$. Similar to shell 0 and shell 1 , the phase shift of the first point toggles between 0 and $\frac{\pi}{p}$. The triangular construction of CQAM points in ring zone 1 is now stated as follows:

Theorem 3: In zone 1 where the the radius is less than or equal to $\sqrt{3}$, CQAM points are determined via the following recursive equations, where $d=2 \sin (\pi / p)$ :

$\rho_{0}=1, \phi_{0}=0$.

$\rho_{1}=\frac{1}{2}\left(\sqrt{4-d^{2}}+\sqrt{3} d\right), \phi_{1}=\frac{\pi}{p}$.

As long as $\rho_{i-1} \leq \sqrt{3}$ :

$\rho_{i}=\rho_{i-2}+d \cdot \sqrt{4-\rho_{i-1}^{2}}$ and $\phi_{i}=\phi_{i-2}$.

For shells outside zone 1, similar reasoning leads to a second limiting radius equal to $\sqrt{7}$. We omit the cumbersome proofs due to space limitation. The second ring zone includes shells with radii in the range $[\sqrt{3}, \sqrt{7}]$. Isosceles triangles defining a new shell $i$ in zone 2 have their bases from two points located at shells $i-1$ and $i-2$ respectively. This creates a rotation shifting the first point away from phase 0 and $\frac{\pi}{p}$ as in zone 1 . The second ring zone is stated by the next theorem with a double recursion on both $\rho_{i}$ and $\phi_{i}$.

Theorem 4: In zone 2 where the the radius is in the range $[\sqrt{3}, \sqrt{7}]$, CQAM points are determined via the following recursive equations, where $d=2 \sin (\pi / p)$ :

Define

$$
\begin{aligned}
\alpha & = \begin{cases}\phi_{i-2}-\phi_{i-1} & \phi_{i-2}>\phi_{i-1} \\
\phi_{i-2}-\phi_{i-1}+\frac{2 \pi}{p} & \text { otherwise }\end{cases} \\
t^{2} & =\rho_{i-1}^{2}+\rho_{i-2}^{2}-2 \rho_{i-1} \rho_{i-2} \cos \alpha .
\end{aligned}
$$

Then as long as $\rho_{i-1} \leq \sqrt{7}$,

$\rho_{i}=\sqrt{d^{2}+\rho_{i-1} \rho_{i-2}\left(\cos \alpha+|\sin \alpha| \sqrt{\frac{4 d^{2}-t^{2}}{t^{2}}}\right)}$

and $\phi_{i}=\phi_{i-1}+\arccos \left(\frac{\rho_{i-1}^{2}+\rho_{i}^{2}-d^{2}}{2 \rho_{i-1} \rho_{i}}\right) \bmod \frac{2 \pi}{p}$.

Finally, beyond radius $\sqrt{7}$, cumbersome equations and isosceles triangles with a corner on shell $i$ (the newly built shell) and two corners from shells $i-2$ and $i-3$ respectively lead to this third theorem. 
Theorem 5: In zone 3 where the radius is in the range $[\sqrt{7}, \sqrt{13}]$, CQAM points are determined via the following recursive equations, where $d=2 \sin (\pi / p)$ :

Define

$$
\begin{aligned}
\alpha & = \begin{cases}\phi_{i-2}-\phi_{i-3} & \phi_{i-2}>\phi_{i-3} \\
\phi_{i-2}-\phi_{i-3}+\frac{2 \pi}{p} & \text { otherwise, }\end{cases} \\
t^{2} & =\rho_{i-2}^{2}+\rho_{i-3}^{2}-2 \rho_{i-2} \rho_{i-3} \cos \alpha .
\end{aligned}
$$

Then as long as $i<p$,

$$
\begin{aligned}
& \rho_{i}=\sqrt{d^{2}+\rho_{i-2} \rho_{i-3}\left(\cos \alpha+|\sin \alpha| \sqrt{\frac{4 d^{2}-t^{2}}{t^{2}}}\right)} \\
& \text { and } \phi_{i}=\phi_{i-3}+\arccos \left(\frac{\rho_{i-3}^{2}+\rho_{i}^{2}-d^{2}}{2 \rho_{i-3} \rho_{i}}\right) \bmod \frac{2 \pi}{p}
\end{aligned}
$$

The recursive equations in Theorem 3 followed by Theorems $4 \& 5$ constitute the complete triangular construction of the 2D CQAM constellation with a total of $p^{2}$ points spread over $p$ shells.

It is obvious that the triangular construction is a greedy algorithm. It minimizes $\sum_{k=0}^{i} \rho_{k}^{2}$ when building the $i$-th shell. A full tree search would lead to the minimal $E_{s}$ (unif) while $d=d_{\text {Emin }}=2 \sin (\pi / p)$ is guaranteed. Table I lists the average energy for both constructions. The full tree search is intractable at large $p$. The good news is the quasi-optimality of the triangular construction.

Table I

TRIANGULAR CONSTRUCTION VERSUS FULL TREE SEARCH.

\begin{tabular}{ccc|ccc}
$p$ & $E_{s}(\mathcal{A})$ & $E_{s}\left(\mathcal{A}_{\text {tree }}\right)$ & $p$ & $E_{s}(\mathcal{A})$ & $E_{s}\left(\mathcal{A}_{\text {tree }}\right)$ \\
\hline 8 & 5.980890 & 5.980890 & 20 & 6.622981 & 6.622981 \\
12 & 6.232051 & 6.232051 & 24 & 6.655495 & $\mathbf{6 . 6 5 3 5 0 5}$ \\
16 & 6.525934 & 6.525934 & 28 & 6.728819 & $\mathbf{6 . 7 2 5 5 2 4}$
\end{tabular}

\section{B. $2 D C Q A M$ as subset of the hexagonal lattice}

The term shell used in previous sections to refer to a subset of CQAM with all points on the same circle comes from the theory of point lattices and sphere packing [4]. In $\mathbb{R}^{2}$, it is natural to compare CQAM constellations to the hexagonal lattice $A_{2}$, the latter being the densest lattice in dimension 2 (highest Hermite constant) and has the maximal kissing number too. The Theta series of $A_{2}$ is $1+6 q+6 q^{3}+6 q^{4}+12 q^{7}+6 q^{9}+\ldots$. Notice that all shells have a population multiple of 6 . Besides tiling regular hexagons in $\mathbb{R}^{2}$ or packing spheres by shifting rows, $A_{2}$ is also obtained by tiling equilateral triangles. This is in direct relationship with our triangular CQAM construction, however this $A_{2}$-CQAM connection exists only for a particular value of $p$ as stated by the following proposition.

Proposition 1: $p^{2}$-CQAM constellations with $p=6$ points per shell are subsets of the lattice $A_{2}$. This special CQAM is plotted in Figure 6.

Proof: $A_{2}$ is built by tiling equal-size equilateral triangles and it is organized by shells around the origin with populations multiple of 6 per shell. Taking 36 points from the first five shells of $A_{2}$ leads to the best figure of merit. This corresponds to a $6^{2}$-CQAM with shells 4 and 5 of equal radius and no isosceles triangles in its construction.

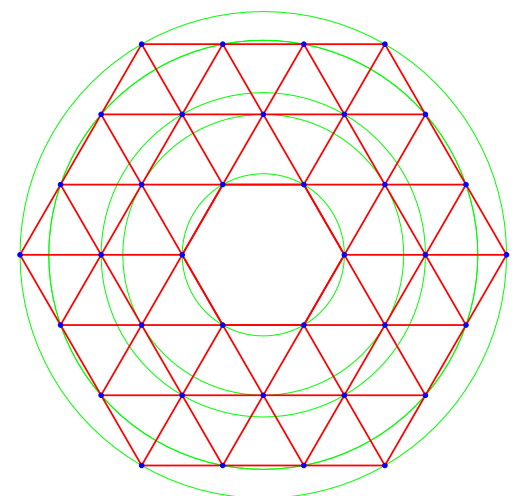

Figure 6. Five shells from the hexagonal lattice coinciding with $6^{2}$-CQAM.

It is possible to construct from $A_{2}$ an extended CQAM with more than 6 shells. Figure 7 shows an extended CQAM with 25 shells and 6 points per shell corresponding to the first 17 shells of the $A_{2}$ lattice.

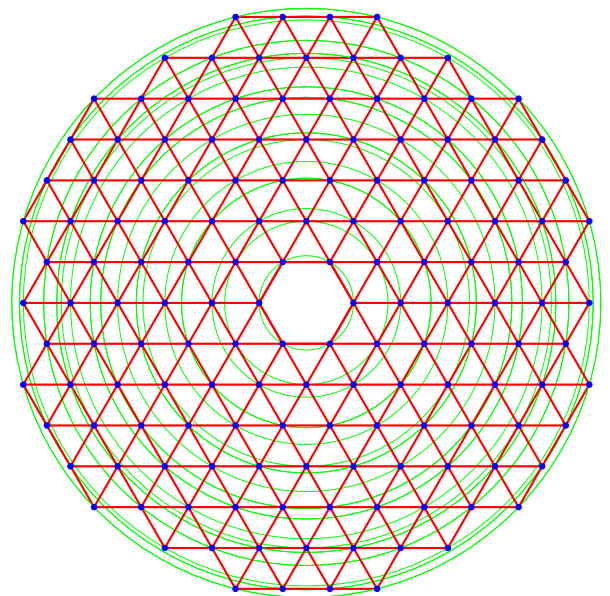

Figure 7. This extended CQAM constellation with 25 shells ( $p=6$ points per shell) is a subset of the hexagonal lattice.

\section{3D CQAM FROM LATTICES AND SPIRALS}

A direct extension of CQAM to 3-dimensional spaces is based on the face-centered cubic lattice $D_{3}$. Similar to the equilateral triangles tiling associated to $A_{2}$, there exists a tiling of the space using a regular polyhedron obtained from two regular tetrahedra and one regular octahedron. The vertices of this tiling form the $D_{3}$ (fcc) lattice. Its Theta series is $1+12 q^{2}+$ $6 q^{4}+24 q^{6}+12 q^{8}+24 q^{10}+8 q^{12}+48 q^{14}+6 q^{16}+36 q^{18} \ldots$ The CQAM triangular construction may coincide with $D_{3}$ if its shells have population multiple of 12 . It is not true for all shells as observed in the Theta series.

Proposition 2: 3D CQAM constellations can be constructed from $D_{3}$ shells with population multiple of 12 .

Proof: We consider building a 3D $p^{2}$-CQAM with 12 points per shell and 12 shells, $p=12$. Take the first 9 shells 
of $D_{3}$ with squared norm varying from 2 to 18 . Drop shells 2,6 , and 8 with squared norms 4,12 , and 16 respectively. Also, drop 12 points out of 36 from the ninth shell. You get 12 shells of a CQAM with a total of 144 points.

The construction of a 3D CQAM with an arbitrary number of points cannot necessarily have a lattice structure. This problem is related to placing $N$ equidistant points on the 3D unit sphere. The number $N$ can be equal to the number $p$ of shells (as in Proposition 2) or we may also consider $N=p^{2}$ so the CQAM cardinality becomes $p^{3}$ points, leading to an uncoded information rate of $\log _{2}(p)$ bits per dimension identical to the rate of a $2 \mathrm{D} p^{2}$-CQAM. In this case we may refer to the constellation as a $p^{3}$-CQAM in $\mathbb{R}^{3}$.

Determining $N$ regularly-placed equidistant points on the 3D unit sphere is only possible for $N=4,6,8,12,20,24$, and 30 points [4]. The vertices, faces, and edges of regular convex polyhedra (the five Platonic solids) make the points of a CQAM shell. Except for those special numbers, this 3D problem has no exact solution. Good methods for placing points almost uniformly on a sphere were proposed in the literature. Besides random methods based on uniform or Gaussian random variables, such as methods published in [5] [10] [8], Fibonacci spirals [15] and generalized spirals [12] on 3D spheres are the best suited to our CQAM construction. However, none of them guarantees a good minimum distance between the points on the spiral. Most of the points in the generalized spiral have better local minimum distance than those on the Fibonacci spiral. Unfortunately, points near the South pole of a generalized spiral are too close.

Minimum distance is improved by considering a new spiral mixing both methods and by slightly moving the first few points near the North pole along the spiral path. Our proposed spiral is a mixture of the Fibonacci spiral and the generalized spiral: in spherical coordinates, $z_{i}$ follows the same expression as a Fibonacci spiral [15] and the phase $\phi_{i}$ follows the formula of a generalized spiral [12]. For $i=0 \ldots N-1$, the $N$ points of our spiral are given by:

$$
z_{i}=1-\frac{2 i+1}{N}, \quad \phi_{i}=\phi_{i-1}+\frac{3.6}{\sqrt{N\left(1-z_{i}^{2}\right)}},
$$

where $\phi_{0}=\phi_{N-1}=0$. For the purpose of illustration only, Figure 8 shows a 3D spiral with 24 points. In general, as mentioned above, it is recommended to consider $p$ shells with $p^{2}$ points per shell for 3D CQAM.

\section{CONCLUSIONS}

Methods for constructing circular QAM constellations in $\mathbb{R}^{2}$ and $\mathbb{R}^{3}$ were presented in this paper. Shaping a signal constellation appears to be an easy task in practice. Numerical values of mutual information (not shown in this document) are very close to capacity. In our next step, the difficulty could be encountered in selecting a suitable $p$-ary error-correcting code with a reasonable complexity that performs well in conjunction with the constellation labeling.

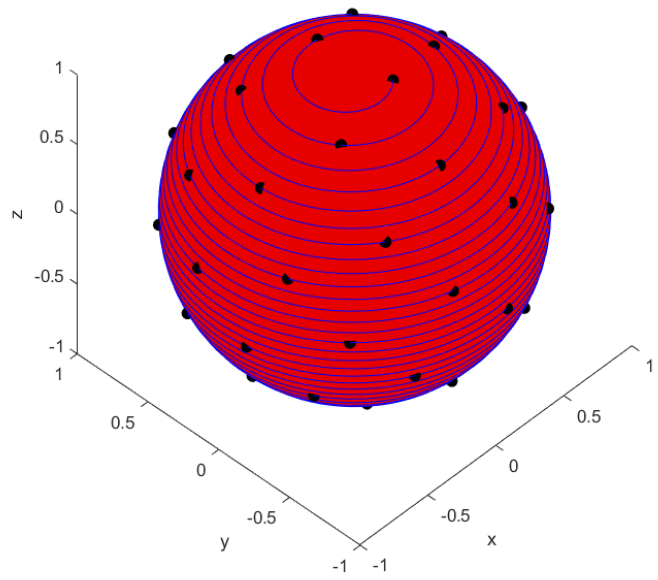

Figure 8. Our spiral for $N=24$ points. The points are almost uniformly placed on the sphere in order to define a CQAM shell.

\section{ACKNOWLEDGMENT}

Johannes Van Wonterghem would like to thank the Research Foundation in Flanders (FWO) for funding his PhD fellowship.

\section{REFERENCES}

[1] G. Böcherer, F. Steiner, and P. Schulte, "Bandwidth efficient and ratematched low-density parity-check coded modulation," IEEE Trans. Commun., vol. 63, no. 12, pp. 4651-4665, Dec. 2015.

[2] J.J. Boutros, F. Jardel, and C. Méasson, "Probabilistic shaping and nonbinary codes," in Proc. IEEE International Symp. on Inf. Theory (ISIT), pp. 2308-2312, Aachen, June 2017.

[3] J.J. Boutros, U. Erez, J. Van Wonterghem, G.I. Shamir, and G. Zémor, "Geometric shaping: low-density coding of Gaussian-like constellations," to appear in Proc. IEEE Inf. Theory Workshop, Guangzhou, China, Nov. 2018.

[4] J. Conway and N.J.A. Sloane, Sphere packings, lattices and groups, 3rd ed., New York (NY), USA: Springer-Verlag, 1999.

[5] J.M. Cook, "Rational Formulae for the Production of a Spherically Symmetric Probability Distribution," Math. of Computation, vol. 11, pp. 81-82, 1957.

[6] N. di Pietro and J.J. Boutros, "Leech constellations of ConstructionA lattices," IEEE Trans. Commun., vol. 65, no. 11, pp. 4622-4631, Nov. 2017

[7] F.R. Kschischang and S. Pasupathy, "Optimal nonuniform signaling for Gaussian channels," IEEE Trans. Inf. Theory, vol. 39, no. 3, pp. 913-929, May 1993.

[8] G. Marsaglia,"Choosing a Point from the Surface of a Sphere," Ann. of Math. Statistics, vol. 43, no. 2, pp. 645-646, 1972.

[9] H. Méric, "Approaching the Gaussian channel capacity with APSK constellations," IEEE Communication Letters, vol. 19, no. 7, pp. 11251128, May 2015

[10] M.E. Muller, "A Note on a Method for Generating Points Uniformly on N-Dimensional Spheres," Communications of the ACM, vol. 2, no. 4, pp. 19-20, Apr. 1959.

[11] J.G. Proakis and M. Salehi, Digital communications, 5th ed., New York (NY), USA: McGraw-Hill, 2008.

[12] E.B. Saff and A.B.J. Kuijlaars, "Distributing many points on a sphere," The Mathematical Intelligencer, Springer, vol. 19, no. 1, pp. 5-11, Dec. 1997.

[13] F. Steiner, G. Böcherer, and G. Liva, "Bit-Metric Decoding of NonBinary LDPC Codes with Probabilistic Amplitude Shaping," to appear in IEEE Communication Letters, 2018.

[14] F.W. Sun and H.C.A. van Tilborg, "Approaching capacity by equiprobable signaling on the Gaussian channel," IEEE Trans. Inf. Theory, vol. 39, no. 5, pp. 1714-1716, Sep. 1993.

[15] R. Swinbank and R.J. Purser, "Fibonacci Grids: A Novel Approach to Global Modelling," Q.J. Royal Meteorological Society, vol. 132, no. 169 , pp. 1769-1793, Feb. 2006. 